\title{
Induced Anticlinic Ordering and Nanophase Segregation of Bow-Shaped Molecules in a Smectic Solvent
}

\author{
Prabal K. Maiti, Yves Lansac, Matthew A. Glaser and Noel A. Clark \\ Department of Physics and \\ Ferroelectric Liquid Crystal Material Research Center, \\ University of Colorado, Boulder, CO 80309
}

\begin{abstract}
Recent experiments indicate that doping low concentrations of bent-core molecules into calamitic smectic solvents can induce anticlinic and biaxial smectic phases. We have carried out Monte Carlo (MC) simulations of mixtures of rodlike molecules (hard spherocylinders with length/breadth ratio $L_{\text {rod }} / D=5$ ) and bow- or banana-shaped molecules (hard spherocylinder dimers with length/breadth ratio $L_{\text {ban }} / D=5$ or 2.5 and opening angle $\psi$ ) to probe the molecular-scale organization and phase behavior of rod/banana mixtures. We find that a low concentration $(3 \%)$ of $L_{\mathrm{ban}} / D=5$ dimers induces anticlinic $\left(\mathrm{SmC}_{A}\right)$ ordering in an untilted smectic $(\mathrm{SmA})$ phase for $100^{\circ} \leq \psi<150^{\circ}$. For smaller $\psi$, half of each bow-shaped molecule is nanophase segregated between smectic layers, and the smectic layers are untilted. For $L_{\mathrm{ban}} / D=2.5$, no tilted phases are induced. However, with decreasing $\psi$ we observe a sharp transition from intralamellar nanophase segregation (bow-shaped molecules segregated within smectic layers) to interlamellar nanophase segregation (bow-shaped molecules concentrated between smectic layers) near $\psi=130^{\circ}$. These results demonstrate that purely entropic effects can lead to surprisingly complex behavior in rod/banana mixtures.
\end{abstract}

PACS numbers: 61.30.-v, 61.30.Cz

Liquid crystals (LCs) are exotic solvents that impose orientational and/or positional ordering on solutes. Conversely, LC ordering and properties are highly sensitive to the influence of low-symmetry solutes. For example, if a chiral solute is added to a nematic a uniform helical twist of the director is induced [1]. By properly choosing the chiral solute the helical twisting power and hence the helical pitch can be controlled and this has potential application in electro-optic displays. If the starting achiral mesophase is $\mathrm{SmC}$, chiral solutes induce the chiral $\mathrm{SmC}^{*}$ phase which is ferroelectric [2,3]. The induced ferroelectric $\mathrm{SmC}^{*}$ has variety of technological applications [4]. Addition of organic solvent in SmA and SmC phase can increase the smectic layer spacing in which solvent is intercalated between smectic layers [5]. Also the smectic ordering can significantly enhance polymerization rate in a monomer-LC mixtures [6]. Results from previous computer simulation studies showed that trans-cis photoisomerization of azo-benzene based LC's in smectic solvent leads to intralamellar nanophase segregation [7]. All these phenomena clearly demonstrate that by appropriately changing the solute or the liquid crystal solvent, material properties can be manipulated for specific technological application.

In recent years a new class of smectic liquid crystal (SmCP phase) made up of achiral molecules with bent cores has been discovered [8.97. The molecules arrange into macroscopically chiral layers characterized by polar ordering and molecular tilt about layer normal, pro- ducing spontaneous polarization. Recent experimental results indicate that a very low concentration of bowshaped molecules in a smectic solvent can give rise to biaxial $\mathrm{SmA}$ /tilted phase [10]. It has also been shown that the polar properties of some liquid crystals can be changed from ferroelectric (FE) to antiferroelectric (AF) by doping with bow-shaped molecules [11]. However, a clear understanding of all these phenomena at a molecular level, essential for molecular engineering is lacking. For example, to select the suitable solutes shape to obtain desired macroscopic properties. Computer simulation can help us to develop a molecular scale interpretation of experimental results and provide theoretical guidance for further experimental studies. In simulation we can gradually change the molecular shape of the solute or solvent and see how these changes affect the large-scale order of liquid crystal systems.

To investigate the effect of low concentration of bowshaped molecules in a smectic solvent, we have carried out a series of Monte Carlo (MC) simulation in a mixture of rodlike and bow- or banana-shaped molecules Some of the questions we want to address are: what new LC phase can be induced by a low concentration of bowshaped molecules in a calamitic LC solvent; how are the bow-shaped molecules are distributed in a LC solvent? How is the structure of the solvent is modified? Under what conditions does nanophase segregation occur. Our model simulations give valuable insight into some of these questions. To a fair approximation the banana molecules 
with small bent angle can be thought of as behaving like platelike molecules. In that respect our simulation can shed light on the phase behavior of such mixture.

The smectic solvent is made up of rodlike molecules represented by hard spherocylinders of length/breadth ratio $L_{\text {rod }} / D$. The bow-shaped molecules are modeled as spherocylinder dimers of length/breadth ratio $L_{\mathrm{ban}} / D$ with opening angle $\psi$ (figure 11). The model of bowshaped molecules as spherocylinder dimers has been investigated through computer simulation and gives rise to rich phase behavior 12, 13 and captures some of the essential physics of such liquid crystalline system. The idea behind using such hard core model is that liquid crystal phase behavior is largely entropy driven and determined by the hard core repulsion between the liquid crystal mesogens. Hard spherocylinder provides a simple model both in terms of computational ease and theoretical approach.

We have performed MC simulations of rod/banana mixtures under NPT (constant particle number, constant pressure, and constant temperature) ensemble with periodic boundary conditions. The simulated systems contained $N=400$ molecules in a orthorhombic box. The number of rodlike molecules was $N_{\text {rod }}=388$ and the number of banana molecules was $N_{\text {ban }}=12$. The concentration of banana molecules, defined as $c_{\mathrm{ban}}=N_{\mathrm{ban}} / N$, is $3 \%$. Initially, the system was prepared in a $\mathrm{SmA}$ state at high pressure. Each trial MC move consisted of a random displacement and reorientation of a randomly selected rod or banana molecule, with the move accepted according to a Metropolis criterion [14]. Simulations were carried out for two sets of length to breadth ratios for rods and bananas. In the first case, $L_{\mathrm{rod}} / D=5$ and $L_{\mathrm{ban}} / D=5$ (the overall banana length is twice the rod length), while in the second case, $L_{\text {rod }} / D=5$ and $L_{\text {ban }} / D=2.5$ (the overall banana length is equal to the rod length). For convenience we introduce reduced units, in which the reduced pressure $P *$ is defined as $P^{*}=\beta P v_{0}$ and the reduced density as $\rho^{*}=\rho v_{0}$, where $v_{0}$ is the volume of a hard spherocylinder of length $L$ and breadth $D$. For all simulations, the pressure was kept fixed at $P^{*}=11$. This pressure corresponds to the middle of the SmA phase for $L / D=5$ spherocylinders [15].

Starting with an untilted SmA phase, we find that low concentration of $L_{\mathrm{ban}} / D=5$ banana molecules induces anticlinic $\left(\mathrm{SmC}_{A}\right)$ ordering in the smectic host for sufficiently large opening angle $\psi$. This behavior is evident in Figure 2(a), where we plot the anticlinic tilt angle $\theta$ with respect to the layer normal as a function of opening angle $\psi \sqrt{16}$. For $100^{\circ} \leq \psi<150^{\circ}$, the molecules within each layer exhibit a uniform tilt, and the system as a whole develops anticlinic ordering. Over this range of opening angles, the anticlinic tilt angle is approximately related to the opening angle by $\theta=(\pi-\psi) / 2$ (dashed line in Figure 2(a). For $\psi<100^{\circ}$, half of each banana molecule is nanophase segregated between smectic layers, and the layers are untilted. For $\psi>150^{\circ}$, the molecules within each layer are uniformly tilted, but there is no overall anticlinic ordering on average. Thus, we observe a transition from $\mathrm{SmA}$ to $\mathrm{SmC}_{A}$ and back to $\mathrm{SmA}$ with decreasing opening angle. This behavior is apparent in instantaneous configurations from the simulations 3 . In figure 2(b) we have plotted the smectic layer spacing vs. opening angle $\psi$. The layer spacing decreases with decreasing opening angle, indicative of increasing molecular tilt, but increases abruptly for $\psi>100^{\circ}$. Note that the layer spacing at $\psi=90^{\circ}$ is larger than that at $\psi=180^{\circ}$, owing to the nanophase segregation of half of each banana molecule between smectic layers.

We have also performed simulations of a $3 \%$ concentration of $L_{\mathrm{ban}} / D=2.5$ banana molecules in an $L_{\mathrm{rod}} / D=5$ spherocylinder host. In this case no tilted phases are observed (Figure 1(a)), and the system remains in the $\mathrm{SmA}$ phase for all $\psi$. However, for $\psi \leq 130^{\circ}$ banana density profile (Figure 5) exhibits a deep minima near the center of the smectic layer and two peaks on the two sides, which indicate that either half of the banana molecules or the entire molecule are nanophase segregated between the smectic layers (interlamellar nanophase segregation). The end-to-end vector is preferentially oriented to the layer normal by $\sim 45^{\circ}$ with the polar vector (unit the vector contained in the plane of the molecule and passing through the apex of the molecule) orienting themselves parallel to the layer normal (Figure 6). For larger $\psi$ there is a transition from interlamellar to intralamellar nanophase segregation, in which banana molecules are segregated within the smectic layers as reflected by both the rod and banana density profiles peaked at the center of the smectic layer (Figure 5. In this case the end-to-end vector is almost parallel to the layer normal with polar vector being in the plane perpendicular to the layer normal (Figure 6). This transition from intralamellar to interlamellar is clearly seen from the equilibrated snapshots of the systems shown in figure 7. Such transition with decreasing opening angle results in an increase in smectic layer spacing as shown in figure $4(\mathrm{~b})$ ). The same kind of nanophase segregation is observed in transcis photoisomerization of azobenzene in a smectic host [7].

Our simulations demonstrate the entropy-driven induction of anticlinic $\left(\mathrm{SmC}_{A}\right)$ ordering in a calamitic $\mathrm{SmA}$ host by doping with a low concentration of bow-shaped molecules with overall length twice that of rodlike solvent molecules. The tilted smectic phase may be the biaxial smectic phase found in the experiment done by Bangalore group in a similar system [10]. For smaller $L_{\mathrm{ban}} / D$, we observe an entropy-driven transition from intralamellar to interlamellar nanophase segregation of bow-shaped molecules in a mixture of rod and banana molecules. In this case, our simulation results demonstrate an orientational transition as a function of opening angle for banana molecules in a SmA solvent. Our model provides 
a tool which can be useful in molecular engineering, for example by selecting suitable shape (in terms of opening angle and length/breadth ratio) to ensure the desired tilt angle or layer spacing. Future work will involve a more systematic study of the phase behavior and structure of such mixture as a function of $L_{\mathrm{ban}} / D, \psi$ and $c_{\mathrm{ban}}$, and of the limits of miscibility of banana molecules in calamitic solvents.

This work was supported by NSF MRSEC Grant DMR 98-09555.

[1] P. G. de Gennes and J. Prost, in The Physics of Liquid Crystals, 2nd ed. (Oxford University Press, New York, 1993).

[2] W. Kuczynski and H. Stegemeyer, Chem. Phys. Lett.70, 123 (1980).

[3] H. Stegemeyer, R. Meister, H. Hoffmann, A. Sprick and A. Becker, J. Mater. Chem., 5, 2183 (1995).

[4] S. T. Lagerwall, in Ferroelectric and Antiferroelectric Liquid Crystals, (WILEY-VCH, Weinheim, 1999).

[5] T. P. Rieker, Liq. Cryst., 19, 497 (1995).

[6] C. A. Guymon, E. N. Hoggan, N. A. Clark, T. P. Rieker, D. M. Walba and C. N. Bowman, Science, 275, 57 (1997).

[7] Y. Lansac, M. A. Glaser, N. A. Clark, and O. D. Lavrentovich, Nature, 398, 54 (1999).

[8] T. Niori, T. Sekine, J. Watanabe, T. Furukawa, and H. Takezoe, J. Mater. Chem., 6, 1231 (1996).

[9] D.R. Link, G. Natale, R. Shao, J.E. Maclennan, N.A. Clark, E. Körblova, D.M. Walba, Science, 278, 1924 (1997).

[10] R. Pratibha, N. V. Madhusudana, and B. K. Sadashiva, Science, 288, 2184 (2000).

[11] E. Gorecka, M. Nakata, J. Mieczkowski, Y. Takanishi, K. Ishikawa, J. Watanabe, H. Takezoe, S. H. Eichhorn, and T. M. Swager, Phys. Rev. Lett., 85, 2526 (2000).

[12] P. H. Camp, M. P. Allen, and A. J. Masters, J. Chem. Phys., 111, 9871 (1999).

[13] Y. Lansac, P. K. Maiti, M. A. Glaser, and N. A. Clark (unpublished).

[14] M. P. Allen and D. J. Tildesley, Computer Simulation of Liquids, (Oxford, 1987).

[15] S. C. McGrother, D. C. Williamson, and G. Jackson, J. Chem. Phys. 104, 6755 (1996).

[16] We determine the anticlinic tilt angle $\theta$ from the secondrank tensorial orientational order parameter $Q_{\alpha \beta}=$ $\left(1 / N_{\text {rod }}\right) \sum_{j=1}^{N_{\text {rod }}}\left(3 u_{j \alpha} u_{j \beta}-\delta_{\alpha \beta}\right) / 2$, where $\alpha, \beta=x, y, z$, and $\mathbf{u}_{j}$ is a unit vector along the long axis of molecule $j$. The tilt angle is defined as the angle between the largesteigenvalue eigenvector of the time-averaged ordering tensor $\langle\mathbf{Q}\rangle$ and the layer normal. To obtain an order parameter appropriate to the $\mathrm{SmC}_{A}$ phase, in which molecules in alternate layers tilt in opposite direction (anticlinic ordering), molecules in alternate layers are rotated by $180^{\circ}$ about the layer normal in the computation of $\mathbf{Q}$.
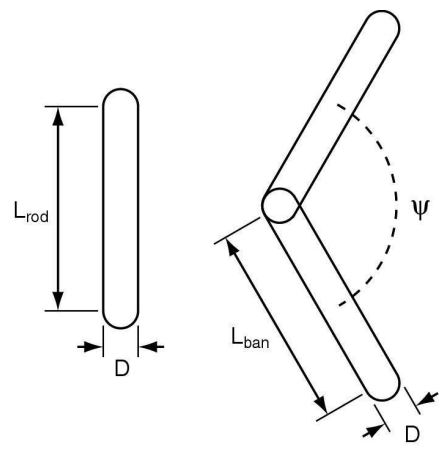

FIG. 1. A schematic representation of model system.

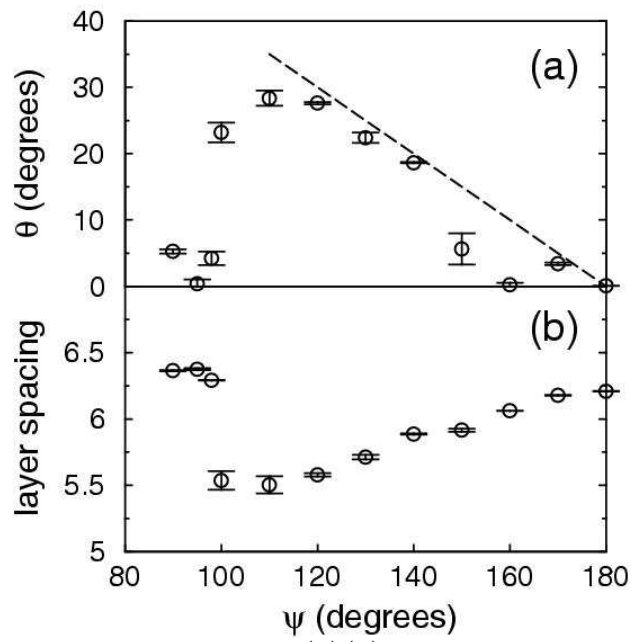

FIG. 2. Induced tilt angle $(\theta)(\mathrm{a})$ and Layer spacing (b) as a function of opening angle $\psi$ for the case when $L_{\text {rod }} / D=5$ and $L_{\text {ban }} / D=5$. 


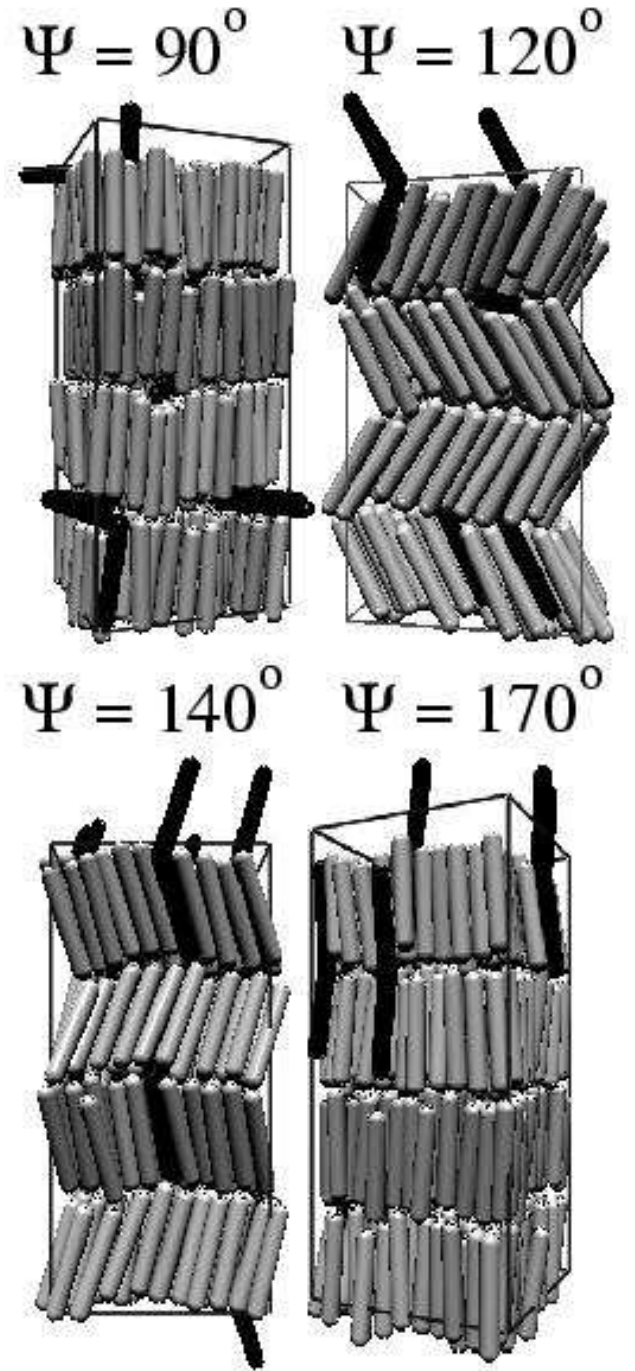

FIG. 3. Equilibrated snapshot of the system for $\psi=90^{\circ}, 120^{\circ}, 140^{\circ}$ and $170^{\circ}$ respectively. The banana molecules are in black and rods are in gray color. The parameters are same as in figure 2 .

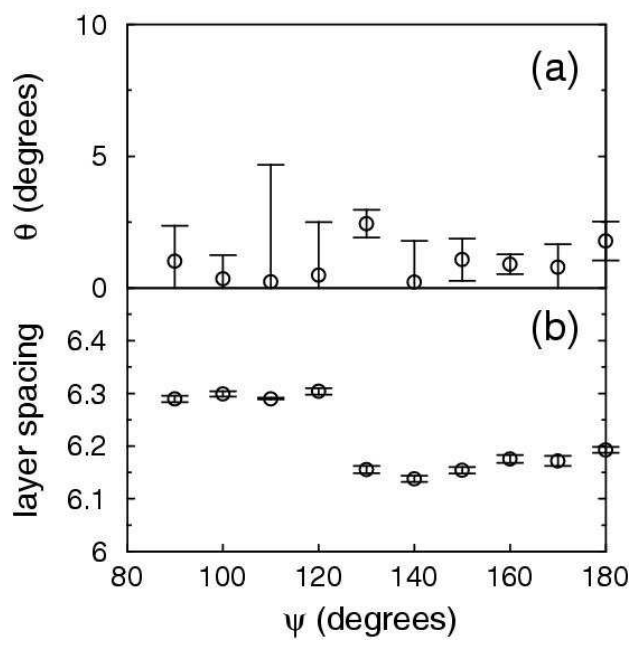

FIG. 4. Tilt angle (a) and smectic layer spacing (b) as a function of the opening angle $\psi$ for the case when $L_{\text {rod }} / D=5$ and $L_{\mathrm{ban}} / D=2.5$.

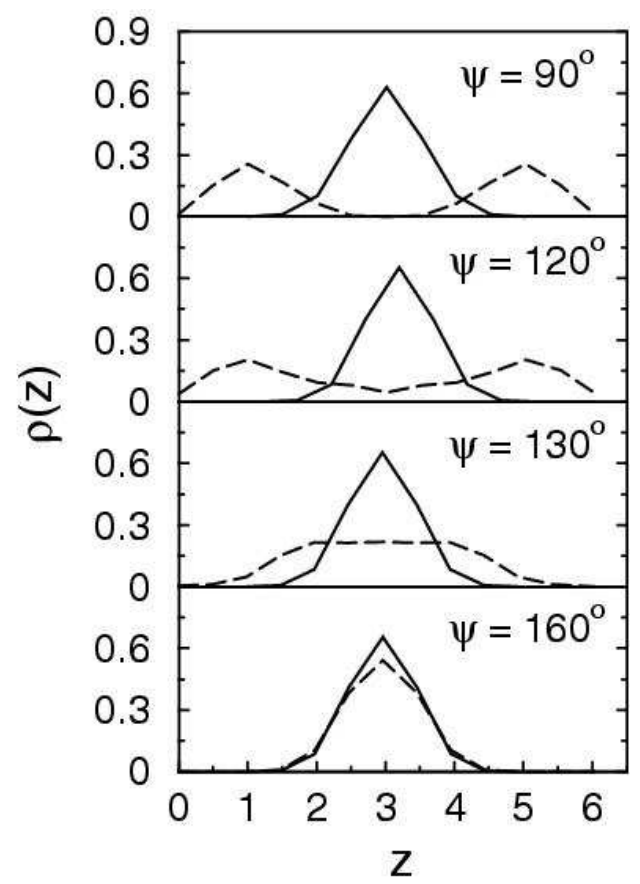

FIG. 5. Density profile for different values of $\psi$ for the case when $L_{\mathrm{rod}} / D=5$ and $L_{\mathrm{ban}} / D=2.5$. Solid line is for rods (solvent) and dashed line for bananas. The banana density has been scaled by 30 for clarity.

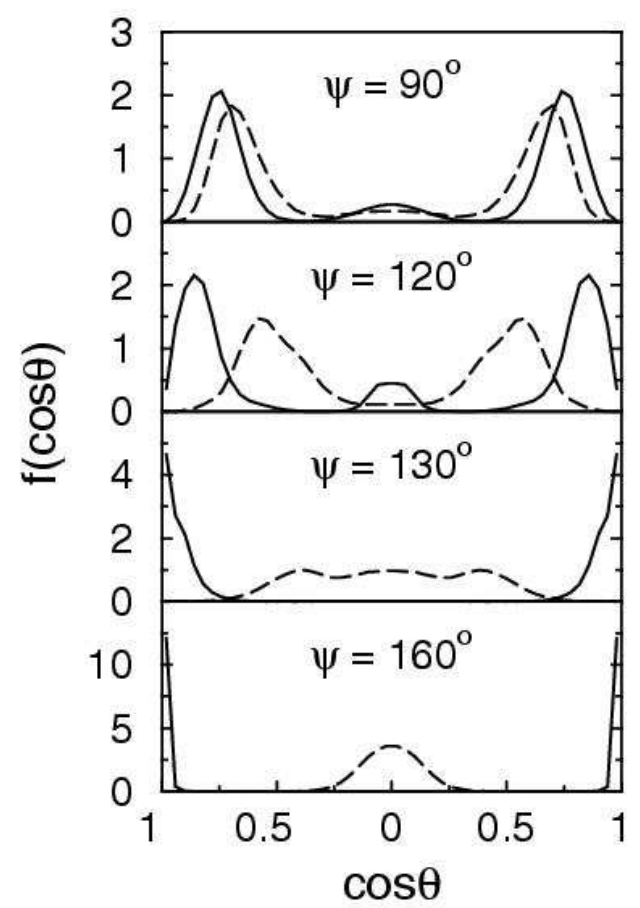

FIG. 6. Orientational distribution of the end-to-end (solid line) and polar vector (dashed line) of the banana molecules for different $\psi$. 

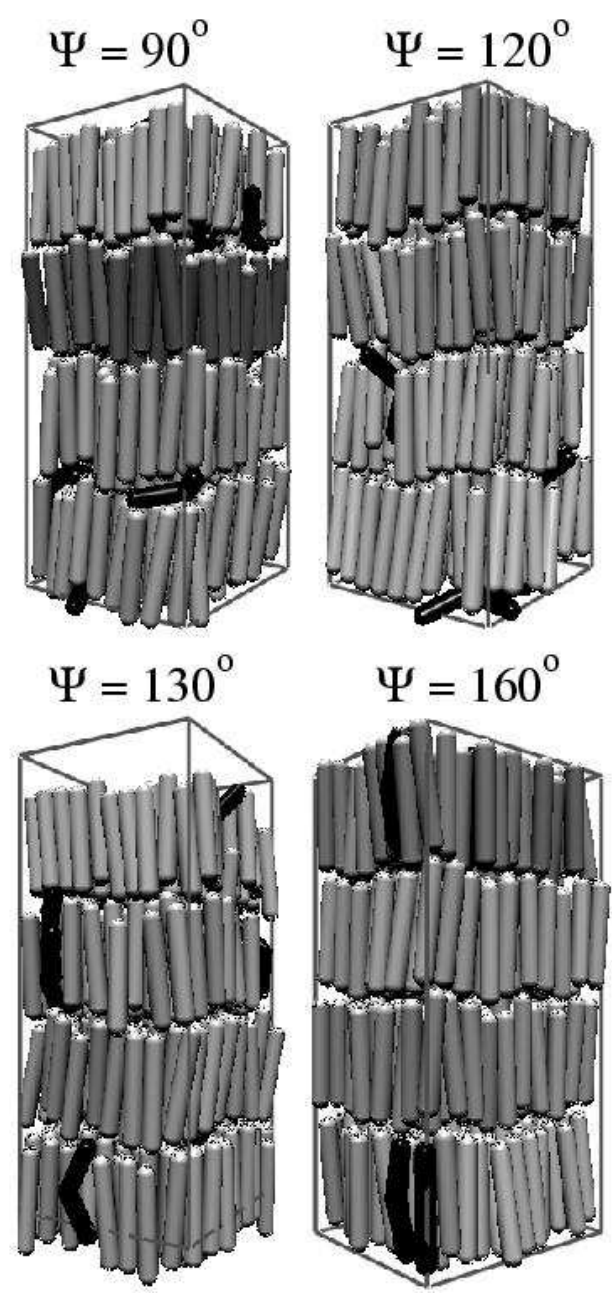

FIG. 7. Equilibrated snapshot of the system for $\psi=90^{\circ}$, $120^{\circ}, 130^{\circ}$ and $160^{\circ}$ respectively. The banana molecules are in black and rods are in gray color. 